CHRONIC OBSTRUCTIVE PULMONARY DISEASE

\title{
Clinical efficacy of anti-pneumococcal vaccination in patients with COPD
}

\author{
I Alfageme, R Vazquez, N Reyes, J Muñoz, A Fernández, M Hernandez, M Merino, \\ J Perez, J Lima
}

See end of article for authors' affiliations

.....................

Correspondence to: Dra I Alfageme,

Pneumology Section, Valme University Hospital, 41014 Seville, Spain; ialfageme@separ.es

Received 2 March 2005 Accepted

28 September 2005

Published Online First

14 October 2005
Background: A study was undertaken to evaluate the clinical efficacy of the 23-valent pneumococcal polysaccharide vaccine (PPV) in immunocompetent patients with chronic obstructive pulmonary disease (COPD).

Methods: A randomised controlled trial was carried out in 596 patients with COPD of mean (SD) age 65.8 (9.7) years, 298 of whom received PPV. The main outcome was radiographically proven community acquired pneumonia (CAP) of pneumococcal or unknown aetiology after a mean period of 979 days (range 20-1454).

Results: There were 58 first episodes of CAP caused by pneumococcus or of unknown aetiology, 25 in the intervention group and 33 in the non-intervention group. Kaplan-Meier survival curves for CAP did not show significant differences between the intervention and non-intervention arms (log rank test $=1.15$, $p=0.28)$ in the whole group of patients. The efficacy of PPV in all patients was $24 \%(95 \% \mathrm{Cl}-24$ to 54 ; $p=0.333)$. In the subgroup aged $<65$ years the efficacy of PPV was $76 \%(95 \% \mathrm{Cl} 20$ to $93 ; p=0.013)$, while in those with severe functional obstruction (forced expiratory volume in 1 second $<40 \%$ ) it was $48 \%$ (95\% Cl -7 to $80 ; p=0.076$ ). In younger patients with severe airflow obstruction the efficacy was $91 \%$ (95\% Cl 35 to 99; $p=0.002)$. There were only five cases of non-bacteraemic pneumococcal CAP, all in the non-intervention group ( $\log$ rank test $=5.03 ; p=0.025$ ). Multivariate analysis gave a hazard ratio for unknown and pneumococcal CAP in the vaccinated group, adjusted for age, of $0.20195 \% \mathrm{Cl} 0.06$ to $0.68 ; p=0.01)$.

Conclusions: PPV is effective in preventing CAP in patients with COPD aged less than 65 years and in those with severe airflow obstruction. No differences were found among the other groups of patients with COPD. ca nfection with Streptococcus pneumoniae remains a major cause of morbidity and mortality across all age groups, being the most common cause of community acquired bacterial pneumonia. ${ }^{1}$ The burden of disease is greatest among young children and the elderly. The incidence of invasive pneumococcal disease has been estimated to be 1530 per 100000 inhabitants per year in developed countries, with the highest rates among persons older than 65 years of age and in children aged under 2 years. In adults, pneumococcal bacteraemia is associated with pneumonia in $60-85 \%$ of cases. ${ }^{2}$ Despite appropriate antibiotic therapy and intensive care treatment, there is a considerable case fatality in pneumococcal bacteraemia-15-20\% among adults overall, with the highest rates among the elderly and in patients with severe underlying medical conditions. ${ }^{3}$ Thus, pneumococcal diseases are a major public health problem all over the world, a problem underlined by the rapidly spreading antimicrobial resistance to common and essential antibiotics.

Smoking is the most common cause of chronic obstructive pulmonary disease (COPD) and the rate of pneumococcal disease is high among patients with this condition, ${ }^{45}$ probably due to defective clearance mechanisms. In these patients, community acquired pneumonia (CAP) is an infectious illness that frequently leads to hospital admission and increased morbidity and mortality. The main aetiology of CAP in these patients is infection due to $S$ pneumoniae in up to $43 \%$ of cases. ${ }^{6}$

The pneumococcal polysaccharide vaccine (PPV) has been found to be effective in the prevention of pneumococcal pneumonia and bacteraemia in previously healthy young people, ${ }^{7}$ and also in certain groups of patients at high risk of developing pneumococcal infection. ${ }^{8}$ However, the degree of protection afforded by PPV remains an issue of some debate, despite the existence of a number of randomised (or quasirandomised) clinical trials, case-control, and indirect cohort studies. The effectiveness of PPV has been questioned for certain populations such as the elderly and some at-risk groups including those with diabetes or chronic respiratory illnesses. Part of the problem is the frequent disparity of results between various clinical trials, ${ }^{9-13}$ including recent meta-analyses that included the 23 serotype PPV. ${ }^{14-16}$

In Spain the 23 serotype PPV was not made commercially available until 1999 and is currently subject to prescription restrictions. It is still not included in general immunisation programmes or recommendations and has been used only to a very limited extent; in practice it is only given to splenectomised patients.

The aim of this study was to evaluate the clinical efficacy of 23 serotype PPV in the prevention of pneumococcal pneumonias in immunocompetent patients with COPD.

\section{METHODS}

Patients were included in the study if they had not been previously vaccinated and had a spirometric diagnosis of COPD at the Pneumology Service of the University Hospital of Valme (in the southern health area of Seville). Patients were

Abbreviations: CAP, community acquired pneumonia; COPD, chronic obstructive pulmonary disease; $\mathrm{FEV}_{1}$, forced expiratory volume in 1 second; PPV, pneumococcal polysaccharide vaccine 
excluded from the study if they were pregnant, were immunosuppressed, or had known neoplasia, renal insufficiency in dialysis, HIV infection, hypogammaglobulinaemia, or anatomical or functional asplenia.

The study was approved by the ethics committee of the hospital. A randomisation code was developed using a computer random number generator in block lengths of 20 ( 10 in each group). Patients who agreed to be included in the study and who fulfilled the selection criteria were informed of the aims and characteristics of the study. They were then randomly assigned to the intervention group and received 23valent pneumococcal capsular polysaccharide vaccine (Pneumo 23; Aventis Pasteur MSD), together with a clinical follow up examination. Subjects assigned to the nonintervention group had a clinical follow up examination but no vaccine. The vaccine was given free of charge at the centre where the patients were recruited. Written informed consent was obtained from all patients.

Demographic, clinical, and spirometric data were taken from each patient at the start of the study. Following inclusion, each patient had a clinical follow up examination over 3 years. The physicians participating in this 3 year follow up were unaware of the group to which individual patients were assigned. The patients were instructed to contact the doctor if they developed a fever of $38^{\circ} \mathrm{C}$ for more than 3 days or if they presented other symptoms that might suggest pneumonia. All the participants were checked routinely every 6 months or if they requested it.

The main outcome measured was time to the first episode of CAP of pneumococcal or unknown aetiology. A diagnosis of pneumonia was based on the finding of a new infiltrate typical of pneumonia which diminished or disappeared during follow up, and symptoms of lower respiratory tract infection with fever. Pneumonia was considered as nosocomial if the onset of symptoms occurred more than 3 days after admission to hospital or a chronic care unit and less than 4 days after discharge. Other cases were considered to have been acquired in the community. A diagnosis of pneumococcal pneumonia was based on the presence of pneumonia and the isolation of $S$ pneumoniae from the sputum (for an adequate sample), bronchoaspirate, blood, pleural fluid, or cerebrospinal fluid. Other aetiological diagnoses were performed according to clinical guidelines. ${ }^{17}$

Patients admitted to hospital were given diagnostic tests as considered necessary by their responsible doctor, according to the seriousness of the case and in order to arrive at a diagnosis (for example, blood culture, thoracocentesis). All patients with an initial diagnosis of pneumonia were subject to a new regime which included clinical revision and radiography 2-4 weeks after the first visit. All the radiographs were examined by two doctors participating in the study.

\section{Analysis of data}

Absolute numbers and percentages were computed to describe the patient population. Medians and quartiles were computed as appropriate. Categorical values were compared using a $\chi^{2}$ or Fisher's test and crude odds ratios with $95 \%$ confidence intervals were computed. Continuous variables

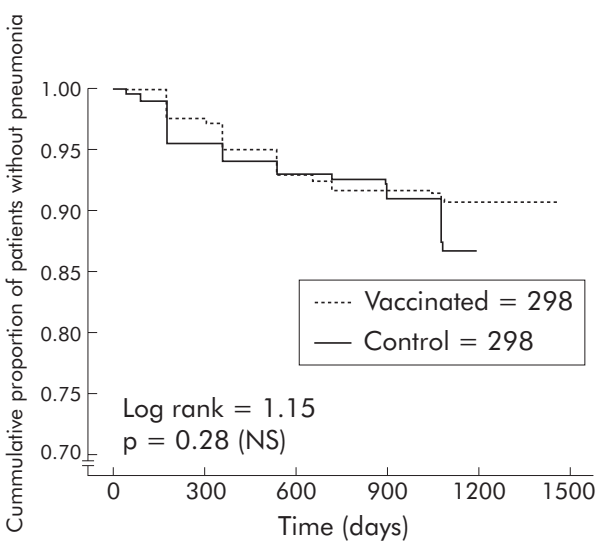

Figure 1 Kaplan-Meier survival curve showing cumulative proportion of patients without pneumonia during the follow up period.

\begin{tabular}{|c|c|c|c|}
\hline & $\begin{array}{l}\text { Intervention group } \\
(\mathrm{N}=298)\end{array}$ & $\begin{array}{l}\text { Non-intervention } \\
\text { group }(\mathrm{N}=298)\end{array}$ & $\mathrm{p}$ value \\
\hline $\begin{array}{l}\% \text { Male } \\
\text { Follow up }\end{array}$ & $96.6 \%$ & $93.3 \%$ & $\begin{array}{l}0.09 \\
0.77\end{array}$ \\
\hline Mean (days) & 980.0 & 977.8 & \\
\hline Range (days) & $20-1454$ & $21-1183$ & \\
\hline Total (person-years) & 800.1 & 798.0 & \\
\hline FVC (I) & $2.0(1.6-2.5)$ & $2.1(1.7-2.6)$ & 0.17 \\
\hline FVC (\%) & $62(50-73)$ & $63(52-74)$ & 0.21 \\
\hline $\mathrm{FEV}_{1}$ (l) & $1.1(0.8-1.4)$ & $1.2(0.8-1.5)$ & 0.02 \\
\hline $\mathrm{FEV}_{1}(\%)$ & $42(32-54)$ & $43(33-55)$ & 0.21 \\
\hline $\mathrm{FEV}_{1} \% \mathrm{FVC}$ & $53(45-62)$ & $55(45-64)$ & 0.21 \\
\hline Age (years) & $69(62-73)$ & $68(61-73)$ & 0.23 \\
\hline \multicolumn{4}{|l|}{ Age group } \\
\hline$<65$ years & $91(31 \%)$ & $116(39 \%)$ & 0.04 \\
\hline$\geqslant 65$ years & $207(69 \%)$ & $182(61 \%)$ & \\
\hline \multicolumn{4}{|l|}{ Severity of COPD } \\
\hline Severe $\left(\mathrm{FEV}_{1}<40 \%\right)$ & 132 (44\%) & $114(38 \%)$ & 0.13 \\
\hline Non-severe $\left(\mathrm{FEV}_{1} \geqslant 40 \%\right.$ ) & $166(56 \%)$ & $184(62 \%)$ & \\
\hline Currently smoking & $65(22 \%)$ & $77(26 \%)$ & 0.25 \\
\hline Previous pneumonia & $58(19.5 \%)$ & $52(17.4 \%)$ & 0.53 \\
\hline Pneumonia in previous 5 years & $33(11 \%)$ & $28(9.4 \%)$ & 0.50 \\
\hline Previous TB & $22(7.4 \%)$ & $33(11.1 \%)$ & 0.12 \\
\hline Neoplasia during follow up & $18(6 \%)$ & $16(5.3 \%)$ & 0.72 \\
\hline Death from any cause & $57(19.1 \%)$ & $58(19.5 \%)$ & 0.92 \\
\hline Death from pneumonia & $6(2 \%)$ & $6(2 \%)$ & 1.00 \\
\hline
\end{tabular}

$\mathrm{FEV}_{1}$, forced expiratory volume in 1 second; FVC, forced vital capacity; TB, tuberculosis. Values are expressed as median (interquartile range) or $\mathrm{n}(\%)$. Two sided $p$ values were calculated using the $\chi^{2}$ test for proportions and the Wilcoxon test for quantitative variables. 
were compared using the two tailed Wilcoxon rank sum test. Unless otherwise stated, statistical significance is indicated by $\mathrm{p}$ values of less than 5\% (two sided). Crude event rates were calculated by dividing the number of cases by the person-time for each outcome. Multivariate Cox proportional hazard models with covariates were used to evaluate the association between the receipt of pneumococcal vaccine and the time to a first outcome event during the study period and excluding repeated episodes from analysis. To investigate the possible effect of age and pulmonary function on the relative risk of pneumonia over time, we included age $(<$ or $\geqslant 65$ years) and the presence of severe airflow obstruction (defined as forced expiratory volume in l second $\left(\mathrm{FEV}_{1}\right)$ $<40 \%$ of expected following SEPAR guidelines ${ }^{18}$ ) as covariates in a Cox regression model, and also an estimation of a possible age $\times$ treatment interaction effect. The effects of the vaccine were therefore investigated in the whole group of 596 patients as well in the subgroups of patients defined by the previously mentioned variables (age and severity of obstruction). The subgroup analysis was performed because of previously published data suggesting that younger patients $\left(<65\right.$ years) and those with more severe obstruction $\left(\mathrm{FEV}_{1}\right.$ $<40 \%$ ) would benefit most from vaccine administration. ${ }^{7} 1216$ Survival curves for patients younger than 65 years and with severe COPD were calculated by the Kaplan-Meier method. The effectiveness of the anti-pneumococcal vaccine was calculated by considering the first episode of pneumococcal pneumonia as the first episode of CAP of unknown aetiology or with isolation of pneumococcus (as $1-R R \times 100)$. The number necessary to treat was calculated as the inverse of the absolute reduction of risk.

The odds ratios and relative risk with $95 \%$ confidence intervals were calculated using EPI-Info (CDC, USA). The remaining tests were performed using SPSS Version 12.0 for Windows.

\section{RESULTS}

Six hundred patients with a diagnosis of COPD were included in the study using progressive intakes. Three hundred received the 23 -valent pneumococcal vaccine and the other 300 were followed clinically during the same period. Four patients (two from each arm of the study) were lost to follow up and were excluded from the final analyses. The study started in October 1999 and was completed in July 2004, which allowed for a minimum follow up of 3 years for each patient except 115 who died before the end of the follow up period. No patient reported any local or systemic reaction to the vaccine. The epidemiological and clinical characteristics of the patients in the two groups are shown in table 1. Although immunosuppressed patients were excluded, neoplasia was detected in 34 patients during follow up, but this finding did not influence the effectiveness of PPV or the incidence of pneumonia.

\section{Pneumonias}

Seventy five patients had an episode of pneumonia during the study, $38(12.7 \%)$ in the intervention group and 37 $(12.4 \%)$ in the non-intervention group. There were a total of 88 episodes of pneumonia, 43 in the intervention group and 45 in the non-intervention group. Sixty four patients had just one episode of pneumonia ( 34 and 30 in the intervention and non-intervention groups, respectively), nine had two episodes (three and six in the intervention and non-intervention groups, respectively), and two had three episodes (one from each group).

Of the 88 episodes of pneumonia, 73 (83\%) were treated in hospital and the other 15 were treated as outpatients. These patients did not receive any special intervention and the doctor responsible for their care decided on the studies to be undertaken to determine the aetiology and treatment. An aetiological diagnosis was obtained for 23 cases; 14 were found to be due to Gram negative bacilli, two to fungi (Aspergillus and Nocardia), two to Staphylococcus aureus, and five to pneumococcus. The remaining 65 had unknown aetiologies. There were 12 nosocomial pneumonias, eight of known aetiology that did not include pneumococcus and four of unknown aetiology. There were 67 first episodes of CAP, 33 in the intervention group and 34 in the non-intervention group. Of these, 58 were either due to pneumococcus or of unknown aetiology (25 in the intervention group, 33 in the nonintervention group). Most of these episodes required hospital admission: $19 / 25(76 \%)$ in the intervention group and $27 / 33$ $(81 \%)$ in the control group $(p=0.59)$. The median length of stay was lower among vaccinated patients, although the differences did not reach statistical significance 9.5 (6.517) days $v 12.0(8.0-20.0)$ days, $p=0.16)$. The total number of days in hospital due to CAP was 242 in the intervention group and 412 in the control group.

\section{Pneumococcal pneumonia}

Streptococcus pneumoniae was isolated from five patients with pneumonia in the non-intervention group and from none in the intervention group ( $p=0.03$, single sided Fisher's test). In three cases it was the only isolated organism, in one Haemophilus influenzae was also isolated, and the remaining patient was also infected with Pseudomonas aeruginosa. Pneumococcus was isolated from the sputum in two cases and from the bronchial secretions obtained by bronchoscopy in the other three. There were no cases of bacteraemic pneumococcal infection.

\section{Time to the first episode of CAP}

Kaplan-Meier survival curves for CAP did not show a significant difference between the intervention and nonintervention arms $(\log$ rank test $=1.15, \mathrm{p}=0.28$, fig $\mathrm{l})$ in the whole group of 596 patients, although there were significant differences in predefined subgroups of patients (see below).

\begin{tabular}{|c|c|c|c|}
\hline & CAP & Persons/year & $\begin{array}{l}\text { Rate }(\times 1000 \text { COPD } \\
\text { cases per year) }\end{array}$ \\
\hline Overall & 76 & 1597.3 & 47.6 \\
\hline Intervention group & 37 & 798.6 & 46.3 \\
\hline Non-intervention group & 39 & 795.7 & 49.0 \\
\hline$<65$ years & 23 & 569.3 & 40.4 \\
\hline$\geqslant 65$ years & 53 & 1026.9 & 51.6 \\
\hline $\mathrm{FEV}_{1}<40 \%$ & 41 & 644.5 & 63.6 \\
\hline $\mathrm{FEV}_{1} \geqslant 40 \%$ & 35 & 952.0 & 36.8 \\
\hline
\end{tabular}


Table 3 Numbers of pneumonia patients (first episode) in a prospective randomised trial showing efficacy of 23-serotype pneumococcal vaccine

\begin{tabular}{|c|c|c|c|c|c|c|c|c|c|}
\hline & \multicolumn{2}{|c|}{ Intervention group } & \multicolumn{2}{|c|}{ Non-intervention group } & \multicolumn{2}{|c|}{ Vaccine efficacy* } & \multirow[b]{2}{*}{ p valuet } & \multicolumn{2}{|c|}{ NNT } \\
\hline & $\mathbf{n}$ & $\mathbf{N}$ & $n$ & $\mathbf{N}$ & $\%$ & $95 \% \mathrm{Cl}$ & & $n$ & $95 \% \mathrm{Cl}$ \\
\hline \multicolumn{10}{|l|}{ CAP-PN } \\
\hline All patients & 25 & 298 & 33 & 298 & 24 & $(-24$ to 54$)$ & 0.333 & & \\
\hline$<65$ years & 3 & 91 & 16 & 116 & 76 & $(20$ to 93$)$ & 0.013 & 10 & (6 to 31$)$ \\
\hline$\geqslant 65$ years & 22 & 207 & 17 & 182 & -14 & $(-107$ to 38$)$ & 0.801 & & \\
\hline $\mathrm{FEV}_{1}<40 \%$ & 12 & 132 & 20 & 114 & 48 & $(-7$ to 80$)$ & 0.076 & & \\
\hline $\mathrm{FEV}_{1} \geqslant 40 \%$ & 13 & 166 & 13 & 184 & -11 & $(-132$ to 47$)$ & 0.945 & & \\
\hline $\begin{array}{l}\text { Age }<65 \text { years and } \\
\text { FEV }<40 \%\end{array}$ & 1 & 46 & 10 & 40 & 91 & (35 to 99) & 0.002 & 3 & $(2$ to 4$)$ \\
\hline Pneumococcal pneumonia & 0 & 298 & 5 & 298 & & & 0.061 & & \\
\hline
\end{tabular}

CAP-PN, community acquired pneumonia of unknown aetiology and pneumococcal pneumonia; FEV 1 , forced expiratory volume in 1 second; NNT, number needed to treat.

*Vaccine efficacy $=1-\mathrm{RR} \times 100$.

†The $p$ value for pneumococcal pneumonia is 0.06 using the Fisher's exact test (two sided) and 0.03 (single sided). This last $p$ value is very similar to that obtained with the log rank test ( $p=0.025$, Kaplan-Meier curves).

\section{Incidence of pneumonia and efficacy of the vaccine}

The incidence of global pneumonia (CAP and nosocomial) in patients with COPD was 55.1 per 1000 patients with COPD per year. In those with CAP, it was 47.6 per 1000 patients with COPD per year (46.3 in the intervention group, 49.0 in the non-intervention group). Table 2 shows the incidence of pneumonia by age and severity of airflow obstruction. The incidence of non-bacteraemic pneumococcal pneumonia was 313 per 100000 patients with COPD per year.

Table 3 shows the results of univariate analysis and the efficacy of the anti-pneumococcal vaccine in preventing pneumonia which, in the whole group of patients with COPD, was $24 \%$ (95\% CI -24 to $54 ; \mathrm{p}=0.333$ ). The efficacy increased in the subgroup of younger patients of $<65$ years up to $76 \%(95 \%$ CI 20 to $93 ; \mathrm{p}=0.013)$ and in those with severe functional obstruction to $48 \%$ (95\% CI -7 to 80 ; $p=0.076)$. In the subgroup of younger patients with severe airflow obstruction the efficacy increased up to $91 \%$ (95\% CI 35 to $99 ; \mathrm{p}=0.002$ ) with a number needed to treat of three COPD patients (95\% CI 2 to 4 ).

The risk ratio (RR) and vaccine efficacy could not be calculated when there was no case of pneumonia with isolated pneumococcus in the intervention group, which is why both groups could only be compared by survival analysis. Accordingly, the Kaplan-Meier survival curves for pneumococcal pneumonia show a significant difference between the intervention and non-intervention groups $(\log$ rank test $=5.03$;

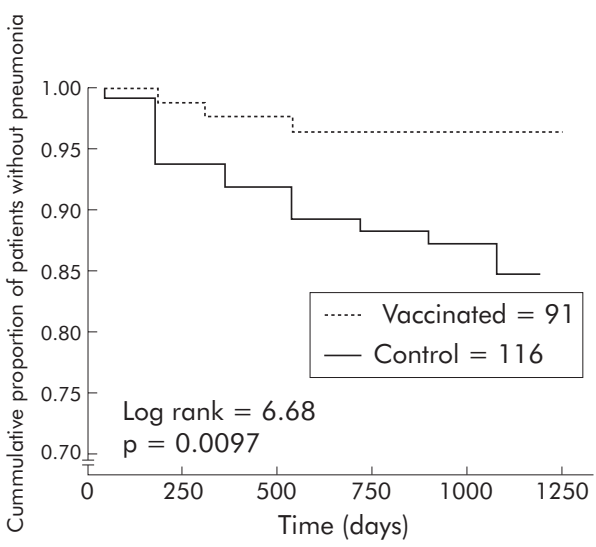

Figure 2 Kaplan-Meier survival curve showing the cumulative proportion of patients $<65$ years without pneumonia during the follow up period. $p=0.0250)$. The Kaplan-Meier curve in fig 2 shows the accumulated percentage of patients $<65$ years without pneumonia (of unknown aetiology and pneumococcus) across time for both the intervention and non-intervention groups (log rank test $=6.68 ; p=0.0097$ ). Figure 3 shows a Kaplan-Meier curve for patients with severe airflow obstruction $\left(\mathrm{FEV}_{1}<40 \%\right.$ predicted), with the accumulated percentage of pneumonias for both groups (log rank test $=3.85 ; p=0.049)$. In the Cox regression multivariate analyses (fig 4 ) the hazard ratio for pneumonia was adjusted for the effects of age $(<65$ or $\geqslant 65$ years, table 4$)$, the severity of obstruction $\left(\mathrm{FEV}_{1}<40 \%\right.$ or $\geqslant 40 \%$ ), and the interaction of age $\times$ vaccine. This interaction means that the efficacy of the vaccine depends on the age of the patients. There was no sign of efficacy in older subjects ( $\geqslant 65$ years) but younger patients ( $<65$ years) in the intervention group were five times less likely to develop pneumonia than a non-vaccinated person of the same age with COPD of the same severity (RR $0.20,95 \%$ CI 0.06 to $0.68 ; \mathrm{p}=0.01$ ). Severity of airflow obstruction $\left(\mathrm{FEV}_{1}<40 \%\right)$ was also a risk factor for developing pneumonia (RR 2.03, 95\% CI 1.21 to 3.41 ; $p=0.008$ ). Similar results could be achieved if the model was stratified by age. Instead of a global model with an interaction term, we obtained two models for ages $<65$ and $\geqslant 65$ years (table 4 ). For younger patients the model was very similar to the previously described global model but, for older patients, there was no indication of vaccine efficacy (RR 1.53, 95\% CI 0.61 to $2.17 ; \mathrm{p}=0.66$ ).

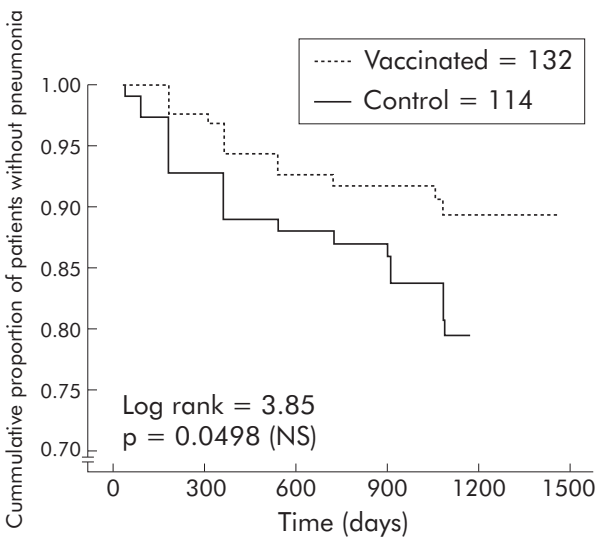

Figure 3 Kaplan-Meier survival curve showing cumulative proportion of patients with severe COPD (FEV $1<40 \%$ ) without pneumonia during the follow up period. 


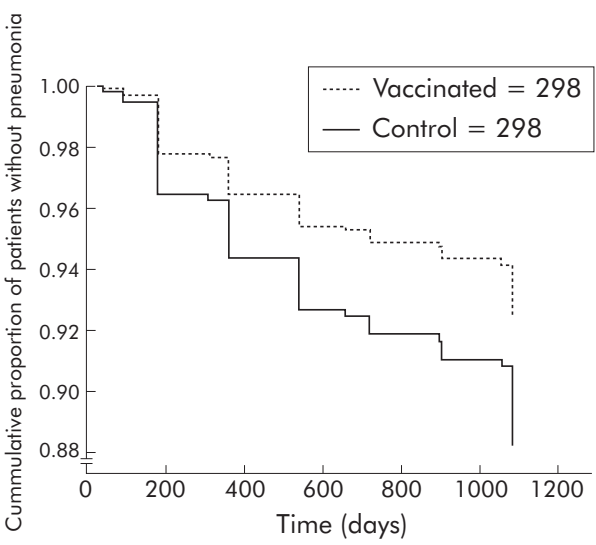

Figure 4 Cox regression model curve showing the proportion of patients with COPD without pneumonia adjusted for age and severity of airflow obstruction during the follow period.

\section{Mortality}

There was no difference in the mortality rates between the two groups which was around 19\%. The major cause of death was respiratory failure in 34 patients, followed by cardiovascular diseases in 29 cases, cancer detected during follow up in 21 subjects ( 11 of these were pulmonary carcinomas), 13 deaths due to infections (12 for pneumonia, one being pneumococcus), 11 to gastrointestinal causes, five to other causes, and two due to unknown causes. Factors influencing the mortality rate among the patients were age (RR 1.05, 95\% CI 1.03 to $1.08 ; \mathrm{p}<0.001$ ), $\mathrm{FEV}_{1} \%$ predicted (RR $0.97,95 \% \mathrm{CI}$ 0.95 to $0.98 ; \mathrm{p}<0.001$ ), current smokers (RR $1.67,95 \% \mathrm{CI}$ 1.08 to $2.60 ; \mathrm{p}=0.022$ ), and the presence of neoplasia (RR $6.54,95 \%$ CI 4.15 to $10.23 ; \mathrm{p}<0.001$ ). The mortality rate for nosocomial pneumonia and CAP in those with COPD was 50.8 per 1000 per year (and 34.4 in those with CAP only).

\section{DISCUSSION}

This study shows that the protective efficacy of 23 serotype PPV in immunocompetent COPD patients for the prevention of radiologically proven pneumonia of pneumococcal and unknown aetiology is linked to two factors-the age of the patient and the severity of the airflow obstruction-as can be seen from the survival analysis. The vaccine had an efficacy of $76 \%$ (95\% CI 20 to 93 ) in patients of $<65$ years of age and $91 \%$ (95\% CI 35 to 99 ) in those who also had severe airflow obstruction. Pneumonia cases from whom pneumococcus was isolated were not found in the intervention group. As expected, cases of pneumococcal bacteraemia were not found in the non-intervention group since bacteraemia occurs in only $10-20 \%$ of patients with pneumococcal pneumonia. ${ }^{27}$
Nevertheless, within this group, the vaccine had no effect in the subgroup of patients over the age of 65 years.

Practically all clinical studies ${ }^{10-12}{ }^{19}$ and meta-analyses ${ }^{7} 15$ agree as to the efficacy of the vaccine in the prevention of invasive pneumococcal disease; however, data regarding the effectiveness of this vaccine in reducing other more common manifestations of pneumococcal disease such as pneumonia have been inconclusive for elderly and high risk groups. Nichol et al, ${ }^{20}$ in a retrospective cohort study, showed that pneumococcal vaccination of elderly people with chronic lung disease was associated with fewer hospital admissions for pneumonia (defined by ICD-9CM codes) and fewer deaths, with the additional effect of influenza vaccination. Hedlund et $a l^{21}$ in a recently published prospective cohort study, showed a reduction in the incidence of hospital admissions for pneumonia and invasive pneumococcal disease in the year following vaccination, adjusted for sex and age. The reasons for these disparate findings include differences in the study population, the prevalence and diagnosis of non-bacteraemic pneumococcal pneumonia, and in the methods used for ascertaining vaccination status. ${ }^{12}$

The incidence of CAP requiring admission to hospital is 2.7 per 1000 of the population, which increases with age to 10.1 per 1000 for those aged over 65 years. ${ }^{22}$ A recent Spanish report established the relative risk of developing pneumonia in patients with COPD as 4.7 times that of the general population over 65 years. ${ }^{23}$ The overall incidence rate of CAP in our COPD patients was 47.6 episodes per 1000 COPD years, which corresponds to that expected. The incidence increased significantly in subjects over the age of 65 years with COPD to 51.6 per 1000 COPD years, and in those with severe airflow obstruction to 63.6 per 1000 COPD years. In this last group the efficacy of the vaccine was shown in the prevention of pneumonias of both pneumococcal and unknown aetiology.

It is highly unlikely that there was any bias towards omitting nosocomial pneumonias of pneumococcal aetiology, since pneumococcus was isolated in 5\% of nosocomial infections from any origin. ${ }^{24}$ Also, we excluded non-pneumococcal cases of known aetiology because mixed infections are produced in about $2 \%$ of cases. ${ }^{22}$ This reduced the number of cases for analysis, but we believe it reflected more closely the true number of non-bacteraemic pneumococcal pneumonias; by contrast, all of the pneumonias were radiologically proven, including those of outpatients. The reduction in pneumonias of unknown aetiology after vaccination supports the suspicion that some of these pneumonias may be due to pneumococcus. Perhaps all of these discrepancies could be clarified with a more widespread use of the antigenuria test to detect pneumococcus. This might identify, in a more precise way, the non-bacteraemic pneumococcal pneumonias. Although the aetiology in our patients was frequently unknown, there were an appreciable number of Gram negative bacterial pneumonias. This aetiology is justified

Table 4 Variables selected by the Cox regression model

\begin{tabular}{|c|c|c|c|}
\hline & $\mathrm{p}$ value & RR & $95 \% \mathrm{Cl}$ \\
\hline \multicolumn{4}{|c|}{ Global model (with interaction between age and vaccine) } \\
\hline Vaccinated $(0=$ no $/ 1=$ yes $)$ & 0.01 & 0.20 & 0.06 to 0.68 \\
\hline Age $(0=<65 / 1=\geqslant 65$ years $)$ & 0.23 & 0.66 & 0.33 to 1.31 \\
\hline Severe airflow obstruction $(0=n o / 1=$ yes $)$ & 0.01 & 2.03 & 1.21 to 3.41 \\
\hline Age $\times$ vaccinated & 0.01 & 5.82 & 1.45 to 23.34 \\
\hline \multicolumn{4}{|l|}{ Model for age $<65$ years } \\
\hline Vaccinated $(0=$ no $/ 1=$ yes $)$ & 0.01 & 0.19 & 0.06 to 0.66 \\
\hline Severe airflow obstruction & 0.04 & 2.62 & 1.04 to 6.55 \\
\hline \multicolumn{4}{|l|}{ Model for age $\geqslant 65$ years } \\
\hline Vaccinated $(0=$ no $/ 1=$ yes $)$ & 0.66 & 1.53 & 0.61 to 2.17 \\
\hline Severe airflow obstruction & 0.07 & 1.81 & 0.96 to 3.39 \\
\hline
\end{tabular}


because many of them were hospital acquired and these patients-because of their more serious condition-have more diagnostic tests performed in the aetiological diagnosis.

Despite many studies of the effectiveness of anti-pneumococcal vaccine for different populations, few have been performed on patients with COPD. Leech et al administered a 14-serotype anti-pneumococcal vaccine to patients with an $\mathrm{FEV}_{1}$ of less than 1.5 litres. No differences were found between the groups in the 2 year follow up period in terms of death or deteriorating conditions, which is attributed to the small number of patients included in the series and the low rate of pneumococcal bacteraemia. Franzen et $a l^{25}$ in another short series study of 65 patients with longstanding emphysema and/or bronchitis found a reduction in the number of pneumonias at 1 year follow up compared with the year before vaccination. The most representative study is the retrospective study of Nichol et $a l^{20}$ involving 1898 patients with an ICD-9-CM diagnosis of chronic lung disease including illnesses other than COPD such as bronchial asthma, bronchiectasis and fibrosis, giving a population less homogeneous than ours.

Our study analysed the efficacy of the anti-pneumococcal vaccine in a homogeneous cohort of patients with a clinical and spirometric diagnosis of COPD. We established the differences in the incidence of pneumonia according to the degree of airflow obstruction in patients with COPD. The incidence of pneumonia was 1.7 times greater in patients with a severe degree of airflow obstruction than in those with light to moderate disease. Analysis of the efficacy of the vaccine according to the degree of airflow obstruction showed that the vaccine tended to be more beneficial in patients with the most severe obstruction. We therefore support the recommendation that anti-pneumococcal vaccine should be administered to these patients, independent of the cost effectiveness analysis already cited..$^{20}$

Most COPD patients with CAP $(76 \%$ and $81 \%$ in the intervention and control groups, respectively) need hospital admission, with prolonged lengths of inpatient stay and increased medical costs. A low cost intervention such as PPV that tends to reduce the incidence of pneumonia should therefore be cost effective.

Nichol $e t a l^{20}$ and Hedlund et $a l^{21}$ found a reduction in the mortality rate of patients who received PPV which can only be explained by a reduction in the specific mortality due to pneumonia of pneumococcal aetiology. However, these studies did not state this categorically, so other uncontrolled factors may have influenced the mortality rate in these patients. In our study the 23 serotype PPV had no effect on the mortality rate. Although it is certain there was a death due to pneumococcal pneumonia in the non-intervention group, this was not sufficient to generate a difference between the groups. The factors that influenced mortality in our patients, in addition to the presence of neoplasia, were those classically described in patients with COPD (age, severity of airflow obstruction, and current smoking).

A considerable limitation of this study is the lack of a blind placebo comparison group. Nevertheless, it is highly unlikely that this limitation significantly influenced the results because the vaccination status of the patient was kept in a specific encrypted database and was not stated in the patients' clinical records. The main investigator of this study (IA) was the only person with access to this database, but this investigator did not participate in the follow up or in adjudicating the outcome events. This task was performed by the physicians conducting the follow up who were unaware of the treatment group allocation of their patients. These investigators were committed not to ask patients about their vaccination status. Furthermore, during the follow up period most patients from both groups also received vaccination against influenza for several years; this could be confusing, especially for old patients from rural areas (the majority in the present study) and probably many of them did not have a clear idea of their anti-pneumococcal vaccination status after 3 years of follow up.

In summary, PPV should be given to patients with COPD aged $<65$ years, especially if they have severe airflow obstruction. This vaccination could prevent episodes of pneumococcal pneumonia frequently labelled as "pneumonia of unknown aetiology". Further studies are needed with a larger sample to examine a possible protective effect in the elderly population and in COPD patients with less severe obstruction. In addition, studies should be undertaken to determine the real incidence of non-bacteraemic pneumococcal pneumonia, as well as perfecting the diagnostic methods used in its detection. This would permit the effectiveness of PPV to be determined with greater precision, and its possible substitution by other types of anti-pneumococcal vaccines, such as pneumococcal protein conjugate vaccine $^{26}$ or by other protein vaccines, to be assessed. ${ }^{27}$

\section{Authors' affiliations \\ I Alfageme, N Reyes, J Muñoz, M Hernandez, M Merino, J Perez, \\ J Lima, Pneumology Section, Valme University Hospital, Seville, Spain R Vazquez, A Fernández, Research Unit, Valme University Hospital, Seville, Spain}

Supported by two grants of SEPAR (Sociedad Española de Neumología y Cirugía Torácica) and SAS (Servicio Andaluz de Salud: 53/99).

Competing interests: none declared.

\section{REFERENCES}

1 Anon. Pneumococcal vaccines. WHO position paper. Wkly Epidemiol Rec 1999;74:177-83.

2 Austrian R, Gold J. Pneumococcal bacteremia with special reference to bacteremic pneumococcal pneumonia. Ann Intern Med 1964;60:759-76.

3 Ortqvist A. Pneumococcal vaccination: current and future issues. Eur Respir J 2001; 18:184-95.

4 Lipsky BA, Boyko EJ, Inui TS, et al. Risk factors for acquiring pneumococcal infections. Arch Intern Med 1986;146:2179-85.

5 Pastor P, Medley F, Murphy TV. Invasive pneumococcal disease in Dallas County, Texas: results from population-based surveillance in 1995. Clin Infect Dis 1998;26:590-5.

6 Torres A, Dorca J, Zalacaín R, et al. Community-acquired pneumonia in chronic obstructive pulmonary disease. A Spanish multicenter study. Am J Respir Crit Care Med 1996;154:1456-61.

7 Fine MJ, Smith MA, Carson CA, et al. Efficacy of pneumococcal vaccination in adults. A meta-analysis of randomized controlled trials. Arch Intern Med 1994; 154:2666-77.

8 Shapiro ED, Berg AT, Austrian R, et al. The protective efficacy of polyvalent pneumococcal polysaccharide vaccine. N Engl J Med 1991;325:1453-60.

9 Leech JA, Gervais A, Ruben FL. Efficacy of pneumococcal vaccine in severe chronic obstructive pulmonary disease. Can Med Assoc J 1987;136:361-5.

10 Koivula I, Sten M, Leinonen $M$, et al. Clinical efficacy of pneumococcal vaccine in the elderly: a randomized, single-blind population-based trial. Am J Med 1997;103:281-90.

11 Ortqvist A, Hedlund J, Burman LA, and Swedish Pneumococcal Vaccination Study Group, et al. Randomised trial of 23-valent pneumococcal capsular polysaccharide vaccine in prevention of pneumonia in middle-aged and elderly people. Lancet 1998;351:399-403.

12 Jackson LA, Neuzil KM, Yu O, et al. Vaccine Safety Datalink. Effectiveness of pneumococcal polysaccharide vaccine in older adults. N Engl J Med 2003;348: 1747-55.

13 Honkanen PO, Keistinen T, Miettinen L, et al. Incremental effectiveness of pneumococcal vaccine on simultaneously administered influenza vaccine in preventing pneumonia and pneumococcal pneumonia among elderly persons aged 65 years or older. Vaccine 1999;17:2493-500.

14 Hutchison BG, Oxman AD, Shannon HS, et al. Clinical effectiveness of pneumococcal vaccine - meta-analysis. Can Family Physician 1999;45:2381-93.

15 Cornu C, Yzebe D, Leophonte $P$, et al. Efficacy of pneumococcal polysaccharide vaccine in immunocompetent adults: a meta-analysis of randomized trials. Vaccine 2001;19:4780-90.

16 Melegaro A, Edmunds WJ. The 23-valent pneumococcal polysaccharide vaccine. Part I. Efficacy of PPV in the elderly: a comparison of meta-analyses, Eur J Epidemiol 2004; 19:353-63.

17 Bartlett JG, Dowell SF, Mandell LA, et al. Practice guidelines for the management of community-acquired pneumonia in adults. Chron Infect Dis 2000;31:347-82. 
18 Barberà JA, Peces-Barba G, Agustí AGN, et al. Guía clínica para el diagnóstico y el tratamiento de la enfermedad pulmonar obstructiva crónica. Arch Bronconeumol 2001;37:297-316.

19 Vila Corcoles A, Ochoa Gondar O, Hospital Guardiola I, et al. Guinea Oruechevarria I, Alvarez Luy M, Grupo de estudio EVAN-65. Efectividad de la vacuna antineumococica en pacientes mayores de 65 años. Medifarm 2003;13:297-304.

20 Nichol KL, Baken L, Wuorenma J, et al. The health and economic benefits associated with pneumococcal vaccination of elderly persons with chronic lung disease. Arch Intern Med 1999;159:2437-42.

21 Hedlund J, Christenson B, Lundbergh P, et al. Effects of a large-scale intervention with influenza and 23-valent pneumococcal vaccines in elderly people: a 1-year follow-up. Vaccine 2003;21:3906-11.

22 Marston BJ, Plouffe JF, File TM Jr, et al. Incidence of community-acquired pneumonia requiring hospitalization. Results of a population-based active surveillance Study in Ohio. The Community-Based Pneumonia Incidence Study Group. Arch Intern Med 1997;157:1709-18.
23 Vila Corcoles A, Ochoa Gondar O, Hospital Guardiola I, et al. La enfermedad pulmonar obstructiva crónica y el tabaquismo: principales factores de riesgo en las neumonías de personas mayores de 65 años. Aten Primaria 2003:31:272.

24 Gonzalez-Romo F, Rubio M, Betriu C, et al. Prevalence and treatment of Gram-positive infections in internal medicine departments of Spanish hospitals: IGP Study. Rev Esp Quimioter 2003; 16:428-35.

25 Franzen D. Clinical efficacy of pneumococcal vaccination - a prospective study in patients with longstanding emphysema and/or bronchitis. Eur J Med Res 2000:5:537-40.

26 Black SB, Shinefield HR, Ling S, et al. Effectiveness of heptavalent pneumococcal conjugate vaccine in children younger than five years of age for prevention of pneumonia. Pediatr Infect Dis $J$ 2002;21:810-5.

27 Briles DE, Hollingshead S, Brooks-Walter A, et al. The potential to use PspA and other pneumococcal proteins to elicit protection against pneumococcal infection. Vaccine 2000;18:1707-11.

\section{LUNG ALERT}

\section{Many asthma deaths may be preventable}

$\Delta$ Harrison B, Stephenson P, Mohan G, et al. An on-going confidential enquiry into asthma deaths in the Eastern region of the UK, 2001-2003. Primary Care Respir J 2005;14:303-13

A Il deaths under the age of 65 with asthma recorded in the first part of the death certificate were examined from a population of approximately 5.25 million people. Hospital and GP records were scrutinised and GPs were interviewed to ensure that asthma was the cause of death. Fifty seven of the original 95 reported deaths were due to asthma; the other 38 deaths were due to $\operatorname{COPD}(n=13)$, cardiac disease $(n=11)$, pneumonia $(\mathrm{n}=9)$, and other conditions.

Of the 57 confirmed asthma deaths, 19 patients had significant co-morbid diseases. 60\% of the asthma fatalities were male, a reverse of the male/female ratio observed in severe asthma. 53\% of patients were felt to have severe asthma according to BTS guidelines, $21 \%$ had moderately severe asthma, 16\% mild asthma, and 11\% not known. Eleven patients (19\%, eight male, eight aged 20 years or less) suffered sudden death from acute severe asthma; 10 of these occurred between April and August suggesting that atopy may have had a role. The other $81 \%$ had more protracted courses of their final illness with potential opportunity for earlier intervention. The authors point out that the data were, by their nature, retrospective and occasionally incomplete.

Patient care was considered appropriate in only 33\% of cases. Failings were present across primary and secondary care and included inadequate follow up (failures to refer to hospital at an appropriate time or to a respiratory specialist once in hospital) and inadequate prescription of inhaled or oral corticosteroids. $81 \%$ of patients had psychosocial or behavioural factors that were considered to be contributory to death. These included poor compliance/failure to attend $(61 \%)$, but also significant smoking (46\%), denial (37\%), depression $(20 \%)$, alcohol abuse $(20 \%)$, and family disharmony (15\%).

The authors endorse the production of an "at risk" register in primary care as advocated by the $2003 \mathrm{BTS} / \mathrm{SIGN}$ asthma guidelines and suggest criteria to guide the construction of such a register.

C Prys-Picard

Clinical Research Fellow, North West Lung Centre, Manchester, UK; cpryspicard@fs1.with.man.ac.uk 